\title{
Canakinumab as monotherapy for treatment of familial Mediterranean fever - first report in Central and Eastern Europe region
}

\author{
Jesenak $\mathrm{M}^{1,2,3}$, Hrubiskova $\mathrm{K}^{4}$, Kapustova $\mathrm{L}^{1}$, Kostkova $\mathrm{M}^{1}$, Banovcin $\mathrm{P}^{1}$ \\ Centre for Periodic Fever Syndromes, Department of Paediatrics, Comenius University in Bratislava, Jessenius \\ Faculty of Medicine in Martin, University Hospital, Martin, Slovakia. jesenak@gmail.com
}

\begin{abstract}
Autoinflammatory disorders (AID) are characterized by spontaneous attacks of acute inflammation with a broad spectrum of clinical symptoms. Ongoing inflammation and reoccurrence of acute flares can lead to the development of amyloidosis. One group of AID is represented by monogenic periodic fever syndromes while familial Mediterranean fever (FMF) is the most common form of AID from this group. Its prevalence in Central and Eastern Europe was reported to be very low. We report a case of FMF patient with a very severe clinical course of FMF and intolerance to colchicine, which is a gold standard for FMF treatment. The clinical effect of the application of anakinra was insufficient and accompanied with side effects and low tolerability. Switching to canakinumab (human monoclonal antibody against IL-1 $\beta$ ) at dose of $150 \mathrm{mg}$ every 4 weeks induced a rapid remission of the disease activity and inflammatory markers. However, due to relapse of acute flares after three weeks from application, the escalation of dose to $300 \mathrm{mg}$ every 4 weeks induced a complete remission of symptoms and significantly improved the quality of life. This is the first report of successful canakinumab administration in FMF patient in Central and Eastern Europe, a region with very low incidence of FMF (Tab. 1, Ref. 16). Text in PDF www.elis.sk. KEY WORDS: autoinflammation, monogenic periodic fever syndromes, familial Mediterranean fever, amyloidosis, colchicine-resistant disease, canakinumab.
\end{abstract}

\section{Introduction}

Autoinflammatory disorders (AIDs) represent an interesting group of genetically inherited immunodeficiency disorders characterized by uncontrolled spontaneous activation of innate immune reactivity. Recurrent uncontrolled onsets of systemic inflammatory response without any evident infectious origin are typically associated with a broad spectrum of clinical symptoms from fever to serositis, arthralgias/arthritis, skin rashes or general fatigue. A specific subgroup of AID is represented by monogenic periodic fever syndromes with an underlying mutation in the genes involved in the regulation of inflammatory cascade $(1,2)$. The spectrum of new AID is expanding, and newly described genetic causes are re-

${ }^{1}$ Department of Paediatrics, Comenius University in Bratislava, Jessenius Faculty of Medicine in Martin, University Hospital, Martin, Slovakia, ${ }^{2}$ Department of Pulmonology and Phthisiology, Comenius University in Bratislava, Jessenius Faculty of Medicine in Martin, University Hospital, Martin, Slovakia, ${ }^{3}$ Department of Clinical Immunology and Allergology, University Hospital, Martin, Slovakia, and ${ }^{4} 5$ th Department of Internal Medicine, Comenius University in Bratislava, Faculty of Medicine, University Hospital Bratislava Ruzinov, Bratislava, Slovakia

Address for correspondence: $\mathrm{M}$. Jesenak, MD, PhD, Department of Paediatrics, Jessenius Faculty of Medicine, Comenius University in Bratislava, Kollarova 2, SK-036 59 Martin, Slovakia.

Phone: +421.43.4203959, Fax: +421.43.4222678

Acknowledgement: The study was supported by the project Measurement of Kinetics of Cilia in Respiratory Tract (ITMS 26220220019), cofinanced by EU sources. ported every year. Familial Mediterranean fever (FMF) is the most common form of monogenic AIDs all over the world with very high incidence in specific geographical areas. On the other hand, the prevalence in Central and Eastern European (CEE) countries was reported to be very low (3). Due to its rare incidence, FMF is usually forgotten to be a part of differential diagnostic algorithms of recurrent fever, and the diagnosis is confirmed with a long-time delay. The late diagnosis of FMF is associated with the development of organ complications, e.g. amyloidosis. There are only few case reports or case report studies published from CEE region, so it is possible that many FMF patients remain unrecognized (3-5).

The standard treatment of FMF patients is colchicine, which is able to decrease the reoccurrence of flares, achieve control over the ongoing spontaneous inflammation and prevent the development of organ amyloidosis. However, $5-10 \%$ of patients are resistant or even intolerant to the treatment with colchicine (6). Since the central role in the spontaneous inflammation flares is played by interleukin $1 \beta$ (IL-1 $\beta$ ), the therapeutic blockade of this cytokine represents another important therapeutic strategy for these patients. Up to now, there are three molecules available on the market, namely anakinra (recombinant antagonist of IL-1 receptor), rilonacept (fully human dimeric fusion protein consisting of extracellular domain of both IL-1 receptor components) and finally, canakinumab (fully human monoclonal antibody against IL-1 $\beta$ ) (Tab. 1) (7). Despite the increasing number of reports about the use of all the three biologicals in the successful management of various periodic fever syndromes, and according to the summary 
Tab. 1. Biologicals against interleukin $1 \beta$ and their selected characteristics (adapted from Moll et al, 2013 (7)).

\begin{tabular}{|c|c|c|c|}
\hline \multirow[b]{2}{*}{ Molecule } & \multicolumn{3}{|c|}{ Interleukin-1 inhibiting biologicals } \\
\hline & Anakinra & Rilonacept & Canakinumab \\
\hline $\begin{array}{l}\text { Characterization } \\
\text { of molecule }\end{array}$ & $\begin{array}{l}\text { Recombinant antagonist } \\
\text { of human IL-1 receptor }\end{array}$ & $\begin{array}{l}\text { Human dimeric fusion protein (extracellular } \\
\text { domain of both IL-1 receptor components) }\end{array}$ & $\begin{array}{l}\text { Fully monoclonal human } \\
\text { antibody against IL-1 } \beta\end{array}$ \\
\hline Target & IL-1 $\alpha$, IL-1 $\beta$ & IL-1 $\alpha$, IL-1 $\beta$, IL-1Ra & IL-1 $\beta$ \\
\hline Biological half-life & 4 hours & 67 hours & 21-28 days \\
\hline Indications & CAPS & CAPS & CAPS, FMF, HIDS, TRAPS \\
\hline Age limitation & none & $\geq 12$ years & none \\
\hline
\end{tabular}

of product characteristics, only canakinumab is indicated for the treatment of FMF.

\section{Clinical study}

We report a case of a 36-year-old man of Caucasian ethnicity with the history of recurrent abdominal pain accompanied with mildly increased fever. His clinical problems started to appear approximately at the age of 17 years. Acute attacks of pain lasted approximately 3 days and were accompanied with the increase in non-specific inflammatory markers (C-reactive protein over 100 $\mathrm{mg} / \mathrm{L}$ ) and negative procalcitonine. Abdominal ultrasound performed during the acute flare did not reveal any pathological findings and no fluid in abdominal cavity was detected. The periods between the pain attacks were accompanied with fatigue, arthralgia and vasculitis-like symptoms on shin. Microbiological examinations were completely negative and did not explain the problems. There was no response to antibiotic treatments prescribed by general practitioners and the analgesics showed only partial clinical response. After three years of duration of the problems, the patient underwent a gastrofibroscopic examination with the findings of Helicobacter pylori-associated antral gastritis, which was treated according to the standard protocols but without any significant effect on the reoccurrence of abdominal pain. The stool was of normal consistence and examination of urine did not yield any pathological finding. A colonoscopic examination showed incipient inflammatory signs following the diagnosis of ulcerative colitis, and a treatment with 5-aminosalicylic acid was initiated. However, neither this therapy improved the clinical status. The control endoscopic examination did not show any pathologic finding in upper and lower parts of gastrointestinal tract. MR-enterography yielded normal results. Thereafter, appendectomy was performed but recurrent abdominal pain persisted. The patient underwent different dietetic interventions, but none succeeded in relief of pain. The serological tests for coeliac disease and stool for faecal calprotectin were negative; porphyria was also excluded. Respiratory symptoms were not present. Four years after the beginning of the symptoms, an intensive back pain emerged besides the abdominal attacks. They responded to subcutaneous applications of morphine only. Family history was negative for any of immune-mediated diseases. No oedemas of the skin, face, or extremities were presented.

Normal levels of immunoglobulins, C3, and C4 components of the complement system, normal antigenic and functional levels of $\mathrm{C} 1$-inhibitor were disclosed by laboratory investigations. Inflam- matory markers returned usually to increased levels between the attacks (C-reactive protein between $7.3-18.9 \mathrm{mg} / \mathrm{L}$ ). Taking into account the recurrent abdominal pain of short duration, negative findings of the previously performed examinations and laboratory testing, and increase in inflammatory markers during abdominal pain, the diagnosis of familiar Mediterranean fever was suggested and genetic analysis performed. The analysis of $M E F V$ gene revealed a pathological missense mutation in exon 10 p.Met694Val (c.2080A $>\mathrm{G}$ ) in a heterozygous state and the disease-associated variant p.Glu148Gln (c.442G $>$ C) in a compound heterozygosity. The examination of serum amyloid A between the flares was 13.2 $-35.8 \mathrm{mg} / \mathrm{L}$. The diagnostic delay between the first symptoms and genetic confirmation of the disease was almost 17 years. The treatment with colchicine was initiated; however, it was withdrawn due to intolerance and side effects (myalgia, acceleration of fatigue, and elevation of creatinine kinase) after two weeks despite its partial effect on the clinical course and symptoms. Subsequently, the application of anakinra, $100 \mathrm{mg}$ per day (body weight $116 \mathrm{~kg}$ ), was started. It led to a partial relief of symptoms and abdominal pain. Despite this treatment, milder abdominal attacks were still present and chronic fatigue together with lower limbs pain and arthralgia persisted. Application of anakinra was accompanied by local reactions and inflammation in the application site. Moreover, severe headache and hypertension (requiring combined antihypertensive therapy) was also present. Serum amyloid A (the highest value 158 $\mathrm{mg} / \mathrm{L}$ ) and C-reactive protein remained elevated despite the application of anakinra and intermittent use of non-steroidal anti-inflammatory drugs. Due to intolerance of colchicine, partial efficacy of anakinra with systemic and local side effects and persistence of chronic fatigue, high frequencies of flares and persistent elevation of serum amyloid, the treatment with canakinumab was started. The initial dose was $150 \mathrm{mg}$ every 4 th week. Surprisingly already after the 1 st dose, the patient reported a significant relief from fatigue and arthralgia; no abdominal pain attack was also noticed within three weeks after the application. Unfortunately, in the 4th week after the first canakinumab application, acute flare reappeared. However, the application of $2 \mathrm{nd}$ dose of $150 \mathrm{mg}$ that followed after 4 weeks, induced disappearance of symptoms and pain. After three weeks, another flare appeared and therefore the escalation of the dose to $300 \mathrm{mg}$ every 4 weeks was introduced. This dose was able to induce a complete remission of symptoms till the next application. The regular application of $300 \mathrm{mg}$ of canakinumab finally led to the achievement of control over the diseases that was expressed with remission of clinical symptoms and normalization of inflammatory 
markers (C-reactive protein less than $5 \mathrm{mg} / 1$ and serum amyloid A $3.69 \mathrm{mg} / \mathrm{L}$ ). The quality of life has significantly improved and patient has become able to return back to work and normal life.

\section{Discussion}

Familial Mediterranean fever is the most common disease out of all monogenic autoinflammatory diseases. The treatment is aimed at the prevention of painful attacks (resulting from serositis) and development of organ amyloidosis. The treatment with non-steroidal anti-inflammatory drugs or corticosteroids could be also used during the acute flare, although it is usually not effective. The gold standard in clinical management of FMF is colchicine with several suggested modes of action (e.g. inhibition of neutrophil chemotaxis through a direct effect on cytoplasmic microtubules, a reduction in the expression of adhesion molecules on white blood cells and endothelial cells etc.) (8). If tolerated well, it is a life-long therapy. It has been used for both adults and children with titration of the applied dose. Its full clinical effect and dose adjustment could be seen usually within several weeks to months after the beginning of its application. Its administration should start once the diagnosis of FMF is confirmed by genetic analysis and must be taken daily, since a missing dose may lead to the attack. In the population with high prevalence of FMF, it can be used also as a diagnostic test, however, in other geographical settings its diagnostic use is not recommended $(1,2)$.

Approximately $5-10 \%$ of patients did not show a sufficient response to colchicine or yielded a significant side effects requiring its withdrawal. Another possibility is the use of biologicals against IL-1 $\beta$ (anakinra, rilonacept, canakinumab). The use of these medicaments is usually indicated in patients with insufficient response to colchicine, those with persistent high serum concentration of amyloid, patients suffering from severe side effects of colchicine therapy, and in cases with associated vasculitis or renal involvement (8). The mode of action of available biologicals differs. Anakinra and rilonacept are targeting especially IL- $1 \alpha$ and IL- $1 \beta$ while anakinra inhibits the receptor for IL-1 and rilonacept neutralises IL-1 in the blood. On the other hand, canakinumab, a fully human monoclonal antibody, binds selectively to IL-1 $\beta$. Canakinumab is the first biological therapy approved by FDA (Food and Drug Administration) and EMEA (European Medicines Agency) for FMF patients (10). Its application leads to rapid remission of symptoms and suppresses the markers of inflammation. Its efficacy and safety were confirmed by case reports, case report series or even doubleblind, randomized, placebo-controlled studies in both children and adults. Clinical response and full remission were observed in the majority of involved patients (11-15). Moreover, in certain cases, the drug administration interval was safely increased during the remission from 4 to 6-8 weeks (15). Several authors recommend for colchicine-resistant FMF patients to start with a short half-life molecule (anakinra) to assess the efficacy of such approach before long half-life IL-1 blocking strategies (canakinumab) are indicated (16).

\section{Conclusions}

Although the gold standard for the treatment of FMF is col- chicine, its narrow therapeutic window makes its application ineffective in a great proportion of patients. Nowadays, fortunately, other alternative treatments can be considered. Our case report confirms an excellent clinical response to canakinumab in FMF patient intolerant to both, colchicine and anakinra. Application of canakinumab should be recommended for patients with Familial Mediterranean fever, who are resistant or intolerant to colchicine or for those with insufficient response to the treatment with colchicine. We would like to increase the awareness of FMF also in regions where low incidence and prevalence are reported.

\section{References}

1. Ozen S, Bilginer Y. A clinical guide to autoinflammatory diseases: familial Mediterranean fever and next-of-kin. Nat Rev Rheumatol 2014; 10: 135-147.

2. Zadeh N, Getzug T, Grody WW. Diagnosis and management of familial Mediterranean fever: integrating medical genetics in a dedicated interdisciplinary clinical. Genet Med 2011; 3: 263-269.

3. Toplak N, Dolezalova P, Costantin T et al. Periodic fever syndromes in Easters and Central European countered: results of a pediatric multinational survey. Pediatr Rhematol 2010; 10: 29.

4. Dallos T, Luksikova Galova L, Macejkova E et al. Familial Mediterranean Fever - First Experiences in Slovakia. Vnitr Lek 2014; 60: 6-11.

5. Sediva A, Horvath R, Manasek $\mathbf{V}$ et al. Cluster of patients with Familial Mediterranean fever and heterozygous carriers of mutations in MEFV gene in the Czech Republic. Clin Genet 2014; 86: 564-569.

6. Gul A. Approach to the patients with inadequate response to colchicine in familial Mediterranean fever. Best Pract Res Clin Rheumatol 2016; 30: 296-303.

7. Moll M, Kuemmerle-Deschner JB. Inflammatory and cytokine blocking strategies in autoinflammatory disorders. Clin Immunol 2013; 147: 242-275.

8. Ozturk MA, Kanbay M, Kasapoglu B et al. Therapeutic approach to familial Mediterranean fever: a review update. Clin Exp Rheumatol 2011; 4: S77-S86.

9. Terreri MTRA, Bernardo WM, Len CA et al. Guidelines for the management and treatment of periodic fever syndromes familiaal Mediterranean fever. Rev Bras Reumatol 2016; 56: 37-43.

10. Ozdogan H, Ugurlu S. Canakinumab for the treatment of familial Mediterranean fever. Expert Rev Clin Immunol 2017; 13: 393-404.

11. Alpa M, Roccatello D. Canakinumab as rescue therapy in familial Mediterranean fever refractory to conventional treatment. Drug Design Dev Ther 2015; 9: 1983-1987.

12. Basaran O, Uncu N, Celikel BA et al. Interleukin-1 targeting treatment in familial Mediterranean fever: an experience of pediatric patients. Mod Rheumatol 2015; 25: 621-624.

13. Gul A, Ozdogan H, Erer B et al. Efficacy and safety of canakinumab in adolescents and adults with colchicine-resistant familial Mediterranean fever. Arthritis Res Ther 2015; 17: 243.

14. Haviv R, Hashkes PJ. Canakinumab investigated for treating familial Mediterranean fever. Expert Opin Biol Ther 2016; 16: 1425-1434.

15. Laskari K, Boura P, Dalekos GN et al. Longterm beneficial effect of canakinumab in colchicine-resistant Familial Mediterranean Fever. J Rheumatol 2017; 44: 102-109.

16. Ter Haar NM, Frenkel J. Treatment of hereditary autoinflammatory diseases. Curr Opin Rheumatol 2014; 26: 252-258.

Received December 16, 2017. Accepted January 3, 2018. 